\title{
Pion Condensation in the Early Universe at Nonvanishing Lepton Flavor Asymmetry and Its Gravitational Wave Signatures
}

\author{
Volodymyr Vovchenko $\odot,{ }^{1}$ Bastian B. Brandt $\odot,{ }^{2}$ Francesca Cuteri $\odot,{ }^{3}$ Gergely Endrődi $\odot,{ }^{2}$ \\ Fazlollah Hajkarim $\oplus^{3,4}$ and Jürgen Schaffner-Bielich $\oplus^{3}$ \\ ${ }^{1}$ Nuclear Science Division, Lawrence Berkeley National Laboratory, 1 Cyclotron Road, Berkeley, California 94720, USA \\ ${ }^{2}$ Fakultät für Physik, Universität Bielefeld, D-33615 Bielefeld, Germany \\ ${ }^{3}$ Institut für Theoretische Physik, Goethe Universität Frankfurt, Max-von-Laue-Strasse 1, D-60438 Frankfurt am Main, Germany \\ ${ }^{4}$ Dipartimento di Fisica e Astronomia, Università degli Studi di Padova, Via Marzolo 8, 35131 Padova, Italy
}

(Received 17 September 2020; revised 2 November 2020; accepted 7 December 2020; published 6 January 2021)

\begin{abstract}
We investigate the possible formation of a Bose-Einstein condensed phase of pions in the early Universe at nonvanishing values of lepton flavor asymmetries. A hadron resonance gas model with pion interactions, based on first-principle lattice QCD simulations at nonzero isospin density, is used to evaluate cosmic trajectories at various values of electron, muon, and tau lepton asymmetries that satisfy the available constraints on the total lepton asymmetry. The cosmic trajectory can pass through the pion condensed phase if the combined electron and muon asymmetry is sufficiently large: $\left|l_{e}+l_{\mu}\right| \gtrsim 0.1$, with little sensitivity to the difference $l_{e}-l_{\mu}$ between the individual flavor asymmetries. Future constraints on the values of the individual lepton flavor asymmetries will thus be able to either confirm or rule out the condensation of pions during the cosmic QCD epoch. We demonstrate that the pion condensed phase leaves an imprint both on the spectrum of primordial gravitational waves and on the mass distribution of primordial black holes at the QCD scale, e.g., the black hole binary of recent LIGO event GW190521 can be formed in that phase.
\end{abstract}

DOI: 10.1103/PhysRevLett.126.012701

Introduction.-The origin of matter-antimatter asymmetry in the Universe is unknown as yet. There are several theoretical attempts to explain this fact which has to originate from the evolution of the very early Universe $[1,2]$. The asymmetry can be expressed in terms of the values of charges that are conserved in the standard model: baryon number $B$, electric charge $Q$, and lepton number $L$. These numbers are conserved during the cosmic evolution following baryo- and leptogenesis [1-4]. Neutrino oscillations start to occur in the early Universe at $T \sim 10 \mathrm{MeV}$, therefore, at higher temperatures not only the total lepton asymmetry is conserved, but also the individual electron, muon, and tau lepton asymmetries. Conservation of these numbers leads to the evolution of chemical potentials of different particles that were present in the thermal bath and contributed to the equation of state of the Universe at early eras.

Recently, the LIGO experiment detected several gravitational wave (GW) events from the merger of black holes predicted by general relativity $[5,6]$. GWs may also have a cosmic origin due to inflation or possible cosmic (phase) transitions [7]. Primordial gravitational waves (PGWs) can

Published by the American Physical Society under the terms of the Creative Commons Attribution 4.0 International license. Further distribution of this work must maintain attribution to the author(s) and the published article's title, journal citation, and DOI. Funded by SCOAP. be produced from the perturbation of spacetime $[8,9]$ by the inflationary phase in the early Universe [10]. Passing through the different stages of cosmic history like the QCD and electroweak transitions, and the matter dominated epoch will leave imprints on PGWs due to the variation of the Hubble expansion rate [11-14].

Black holes (BHs) can either form by the collapse of matter in stars or in the early Universe due to primordial density perturbations generated by inflation $[15,16]$. The latter ones are known as primordial black holes (PBHs) possible dark matter candidates [17]. The formation of PBHs is caused by the collapse of inhomogeneous high density regions during the time the modes cross the horizon [18-20]. These processes depend on the inflationary scenario and the scales adopted, as well as on the thermal history of the early Universe, making them sensitive to the matter-antimatter asymmetry.

For an isentropic expansion of the Universe it is common to express the asymmetries in terms of the conserved charge per entropy ratios: $b=n_{B} / s, q=n_{Q} / s$, and $l_{\alpha}=n_{L_{\alpha}} / s$ with $\alpha=e, \mu, \tau$. One can associate a chemical potential to each of the conserved charges $B, Q$, and $\left\{L_{\alpha}\right\}$. The cosmic trajectory is a line in the six-dimensional space of $T, \mu_{B}, \mu_{Q}$, $\mu_{e}, \mu_{\mu}$, and $\mu_{\tau}$ defined by five conservation equations:

$$
\frac{n_{B}\left(T, \mu_{B}, \mu_{Q}\right)}{s\left(T, \mu_{B}, \mu_{Q},\left\{\mu_{\alpha}\right\}\right)}=b,
$$




$$
\begin{gathered}
\frac{n_{Q}\left(T, \mu_{B}, \mu_{Q},\left\{\mu_{\alpha}\right\}\right)}{s\left(T, \mu_{B}, \mu_{Q},\left\{\mu_{\alpha}\right\}\right)}=0, \\
\frac{n_{L_{\alpha}}\left(T, \mu_{Q},\left\{\mu_{\alpha}\right\}\right)}{s\left(T, \mu_{B}, \mu_{Q},\left\{\mu_{\alpha}\right\}\right)}=l_{\alpha}, \quad \alpha \in e, \mu, \tau .
\end{gathered}
$$

The conserved charge and entropy densities entering the above equations are given as functions of the temperature and chemical potentials through the equation of state of cosmic matter. For the cosmic QCD epoch, the equation of state is mainly determined by strongly interacting matter, but also contains the contributions of leptons and photons. Naturally, nontrivial dynamics is mainly contained in the QCD part.

Tight constraints on the baryon asymmetry and electric charge are available: $b=(8.60 \pm 0.06) \times 10^{-11}$ and $q=0$. The total lepton asymmetry in the standard scenario arises through sphaleron processes, giving $l=-(51 / 28) b$, equally distributed among the three lepton flavors [21]. This yields the standard cosmic trajectory where all chemical potentials are close to vanishing for the majority of the cosmic trajectory. Values of the total lepton asymmetry considerably larger than the baryon one are also possible: Ref. [22] gives the constraint of $|l|<0.012$. Here $l=l_{e}+l_{\mu}+l_{\tau}$. A recent analysis of Ref. [23] shows that pion condensation is unlikely to occur under this constraint if the lepton asymmetry is equally distributed among the three flavors. However, due to the absence of neutrino oscillations at $T \gtrsim 10 \mathrm{MeV}$, the individual lepton flavor asymmetries are not strongly constrained. It has been pointed out in Refs. $[23,24]$ that sufficient conditions for pion condensation to occur can be achieved for unequally distributed lepton asymmetries. Complementary to Ref. [24], in the present Letter we determine these conditions specifically [25]. Moreover, we point out, for the first time, signatures of a pion-condensed phase in the early Universe, namely its impacts on the spectrum of PGWs and on $\mathrm{PBH}$ formation.

Equation of state.-Pion condensation is expected to occur if the electric charge chemical potential $\mu_{Q}$ exceeds the pion mass. First-principle lattice QCD studies at finite isospin density do suggest pion condensation to take place at $T \lesssim 160 \mathrm{MeV}$ and $\mu_{I} \gtrsim m_{\pi}$ [26,27], with $\mu_{I}$ being the isospin chemical potential [28]. Here we analyze the cosmic trajectories determined by Eqs. (1)-(3) at different values of $l_{e}, l_{\mu}$, and $l_{\tau}$ to determine the conditions for pion condensation to occur. Notice that the weak decays of pions are blocked in the present setting of weak equilibrium, since all outgoing neutrino states are filled due to the high lepton chemical potentials, stabilizing the pion condensate [29].

Neglecting QED interactions, the standard model equation of state is partitioned into contributions from QCD, leptons, and photons:

$$
p=p_{\mathrm{QCD}}\left(T, \mu_{B}, \mu_{Q}\right)+p_{L}\left(T, \mu_{Q},\left\{\mu_{\alpha}\right\}\right)+p_{\gamma}(T) .
$$

The leptonic pressure is modeled by an ideal gas of charged leptons and neutrinos, including all three lepton flavors. The photonic pressure is given by a massless ideal gas of photons.

As we focus our study on temperatures $T<160 \mathrm{MeV}$ that are relevant for hadronic matter, the QCD pressure is approximated by a variant of the hadron resonance gas (HRG) model. In the standard HRG model one includes all known hadrons and resonances as free particles. The HRG model provides a reasonable description of the QCD equation of state in this temperature range when compared to the results of first-principle lattice QCD calculations [30,31]. To incorporate the pion-condensed phase we modify the HRG model by replacing the free pion gas by an interacting pion gas, modeled by a quasiparticle (effective mass) approach [32] matched to chiral perturbation theory [33] and lattice QCD results at zero temperature (see details in Ref. [34]). The reliability range of the model is established through comparisons to our first-principle lattice QCD results at $\mu_{I}>0$, as detailed in Ref. [34]. The phase diagram of the model in the $\mu_{Q}-T$ plane is shown in Fig. 1.

The QCD pressure thus consists of the pressure of three pion species, each described by an effective mass model, and by contributions of the rest of the hadrons and resonances that are modeled as free particles:

$p_{\mathrm{QCD}}\left(T, \mu_{B}, \mu_{Q}\right)=\sum_{i \in \pi} p_{i}^{\mathrm{EM}}\left(T, \mu_{i}\right)+\sum_{j} p_{j}^{\mathrm{id}}\left(T, \mu_{j}\right)$.

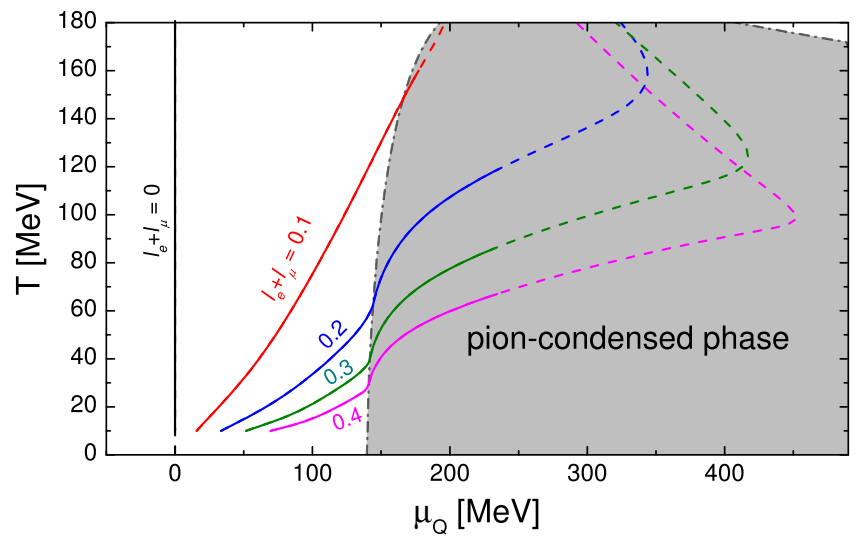

FIG. 1. The phase diagram of an interacting hadron resonance gas with pion condensation in the $\mu_{Q}-T$ plane. The dash-dotted line separates the pion condensed phase (shaded area) from the normal phase. The colored lines depict cosmic trajectories for different values of the lepton flavor asymmetries: the standard cosmic trajectory (black line) and $l_{e}+l_{\mu}$ equal to 0.1 (red line), 0.2 (blue line), 0.3 (green line), and 0.4 (magenta line). In all cases $l_{e}=l_{\mu}$ and $l=l_{e}+l_{\mu}+l_{\tau}=0$. The dashed parts of the trajectories correspond to regions where the effective mass model cannot be reliably validated with the lattice data. 
Here $\mu_{j}=B_{j} \mu_{B}+Q_{j} \mu_{Q}$ with $B_{j}$ and $Q_{j}$ being the baryon and electric charge of hadron species $j$, respectively. The index $i$ sums over the three pion species and the index $j$ sums over all hadrons excluding pions. We include all established light flavored and strange hadrons listed in Particle Data Tables [53].

All the conserved charge densities and the entropy density entering Eqs. (1)-(3) are calculated as the corresponding derivatives of the pressure function [Eq. (4)]: $n_{i}=\partial p / \partial \mu_{i}$ for $i=B, Q, e, \mu, \tau$, and $s=\partial p / \partial T$. For given values of the baryon and lepton asymmetries $b$ and $l_{\alpha}$, we evaluate the cosmic trajectory in the temperature range $10<T<180 \mathrm{MeV}$ by numerically solving Eqs. (1)-(3) for the chemical potentials at each temperature. The numerical solution is achieved using Broyden's method [54]. The procedure is implemented within an extended version of the open source Thermal-FIST package [55]. We tested this procedure by reproducing the cosmic trajectories reported in Ref. [23] using the HRG model.

Cosmic trajectories.-We fix $b=8.6 \times 10^{-11}$ and perform a parametric scan in $l_{e}$ and $l_{\mu}$. As the restriction $|l|<0.012$ on the total lepton asymmetry is rather strong we shall set $l_{\tau}=-\left(l_{e}+l_{\mu}\right)$, meaning that we have a vanishing total lepton asymmetry $(l=0)$ in all our calculations. For each value of $l_{e}$ and $l_{\mu}$, we start calculations at $T=10 \mathrm{MeV}$, where all cosmic trajectories are very similar, and gradually increase the temperature. If the cosmic trajectory enters the phase with a Bose-Einstein condensate of pions, we register the temperature $T_{\text {cond }}$ where the trajectory crosses the pion condensation boundary.

Our calculations reveal that $T_{\text {cond }}$ depends mainly on the sum $l_{e}+l_{\mu}$ of the electron and muon lepton asymmetries, whereas the dependence on the difference $l_{e}-l_{\mu}$ is mild. This is shown in Fig. 2, where we depict the dependence of the temperature $T_{\text {cond }}$ on the sum $l_{e}+l_{\mu}$. The difference $l_{e}-l_{\mu}$ is varied in a range $\left|l_{e}-l_{\mu}\right|<0.5$, giving the narrow black uncertainty band in Fig. 2. At temperatures between $T_{\text {cond }}$ and the chiral crossover pseudocritical temperature $T_{\mathrm{pc}} \approx 160 \mathrm{MeV}$ the cosmic matter is in a pion-condensed

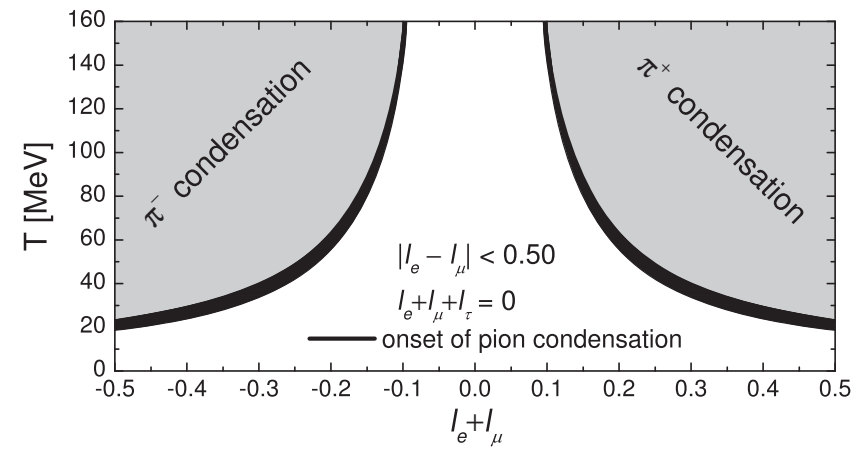

FIG. 2. Dependence of the pion condensation onset temperature on the sum $l_{e}+l_{\mu}$ of electron and muon flavor asymmetries. The bands result from a variation of the difference of electron and muon asymmetries in a range $\left|l_{e}-l_{\mu}\right|<0.50$. phase. We find that pion condensation occurs in the early Universe at $T<160 \mathrm{MeV}$ if the following condition is met:

$$
\left|l_{e}+l_{\mu}\right| \gtrsim 0.1
$$

Pion condensation is not observed at smaller absolute values of $l_{e}+l_{\mu}$. The relation Eq. (6) can therefore be regarded as a universal criterion for pion condensation in the early Universe. Positive values of $l_{e}+l_{\mu}$ correspond to $\pi^{+}$condensation, while negative $l_{e}+l_{\mu}$ imply $\pi^{-}$ condensation.

The temperature dependence of $\mu_{Q}$ is shown in Fig. 1 for several different values of lepton flavor asymmetries in the range $0 \leq l_{e}+l_{\mu} \leq 0.4$. These values are motivated by various theoretical predictions to explain the baryon and lepton asymmetry in the early universe, see Refs. [56-61]. For $l_{e}+l_{\mu}=0$ one essentially recovers the standard cosmological trajectory where $\mu_{Q}$ is very close to zero throughout and far away from the pion condensed phase. For sufficiently large absolute values of $l_{e}+l_{\mu}$ [see Eq. (6)], the cosmic trajectory crosses the pion condensation boundary. The kinklike structure in the cosmic trajectory, predominantly visible for the $l_{e}+l_{\mu}=0.4$ case at $T \approx 95 \mathrm{MeV}$, is associated with a rapid growth of the lepton chemical potentials.

The equation of state exhibits an interesting behavior for trajectories that enter the pion condensed phase. Of particular interest is the interaction measure, $(\varepsilon-3 p) / T^{4}$. The interaction measure is negative deep in the pioncondensed phase at moderate temperatures (see Fig. 3) a distinctive feature of the pion condensed phase also seen in lattice QCD calculations. Figure 3 depicts the temperature dependence of $(\varepsilon-3 p) / T^{4}$ along the cosmic trajectory for the four different cases of positive $l_{e}+l_{\mu}$ values discussed above. The behavior of these two quantities is significantly affected at large lepton asymmetries. For $\left|l_{e}+l_{\mu}\right| \gtrsim 0.3$ the cosmic trajectory passes through a

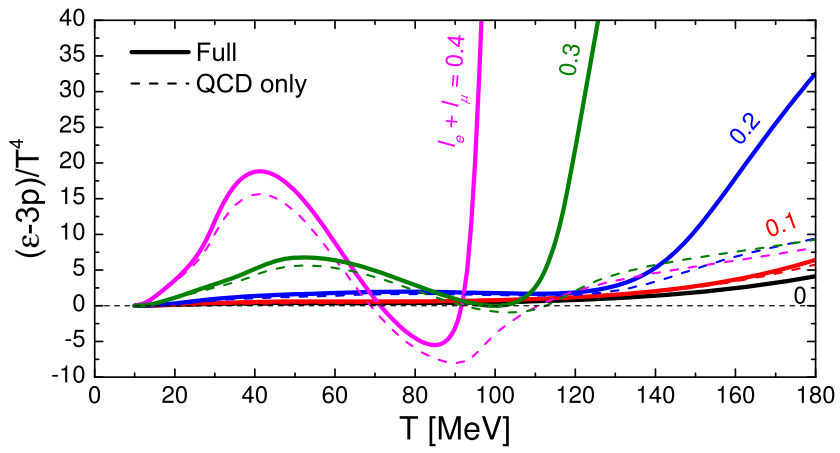

FIG. 3. Temperature dependence of the interaction measure, $(\varepsilon-3 p) / T^{4}$ along the cosmic trajectory for different values of the lepton flavor asymmetries: $l_{e}+l_{\mu}=0$ (black line), 0.1 (red line), 0.2 (blue line), 0.3 (green line), and 0.4 (magenta line). In all cases $l_{e}+l_{\mu}+l_{\tau}=0$. 
region with negative $(\varepsilon-3 p) / T^{4}$, as illustrated by the magenta curve in Fig. 3 for $l_{e}+l_{\mu}=0.4$. Negative interaction measure correlates with large sound velocities that go above the conformal limit of $c_{s}^{2}=1 / 3$. The interaction measure grows to large values $(\varepsilon-3 p) / T^{4} \gtrsim$ 10 at larger temperatures. This drastic rise is a consequence of large lepton chemical potentials at these temperatures, which emerge from lepton flavor number conservation.

Effects on the spectrum of PGWs.-Because of the presence of a nonvanishing lepton asymmetry and the possible formation of the pion-condensed phase, the equation of state before big bang nucleosynthesis (BBN) can change, which will leave an imprint on the PGW spectrum $[13,14,62,63]$.

The evolution of each polarization $\lambda$ of tensor perturbation $h$ for a mode $k$ in cosmology is given by $[10,64]$

$$
h_{\mathbf{k}, \lambda}^{\prime \prime}+2 \frac{a^{\prime}}{a} h_{\mathbf{k}, \lambda}^{\prime}+k^{2} h_{\mathbf{k}, \lambda}=0,
$$

where the $' \equiv d / d \eta$ is the derivative with respect to conformal time $\eta$ and $a$ is the scale factor $(a d \eta=d t, t$ is the cosmic time). The primordial tensor perturbation can be written in terms of the transfer function $X$, tensor perturbation amplitude $h_{\mathbf{k}, \lambda}^{\text {prim }}$, and tensor power spectrum parameterized with respect to a characteristic scale $\tilde{k}=0.05 \mathrm{Mpc}^{-1}$

$h_{\mathbf{k}, \lambda}(\eta) \equiv h_{\mathbf{k}, \lambda}^{\mathrm{prim}} X(k, \eta), \quad \mathcal{P}_{T}=\sum_{\lambda}\left|h_{\mathbf{k}, \lambda}^{\mathrm{prim}}\right|^{2}=A_{T}\left(\frac{k}{\tilde{k}}\right)^{n_{T}}$,

where $A_{T}=r A_{S}$ and $A_{S}, n_{T}$ are scalar and tensor perturbation amplitudes, and the tensor spectral index, respectively. The tensor to scalar ratio denoted by $r$ has an upper limit from measurements by PLANCK of $r \lesssim 0.07[65,66]$.

To compute the temporal evolution of the scale factor one needs to solve the Friedmann equation $\left(H^{2}=(\dot{a} / a)^{2}=\left(8 \pi / 3 M_{\mathrm{Pl}}^{2}\right) \varepsilon, \quad M_{\mathrm{Pl}}=1.22 \times 10^{19} \mathrm{GeV}\right)$. We solve Eq. (7) for a mode $k$ using Eq. (8) until horizon crossing [67], i.e., when $k=|\mathbf{k}|=a\left(\eta_{h}\right) H\left(\eta_{h}\right)$, then we use the WKB (Wentzel, Kramers, Brillouin) approximation for the PGW afterwards until today [11,12]. Using Eqs. (7) and (8) the relic density of PGWs for different frequencies $\nu=k / 2 \pi$ at today $\left(a_{0}\right)$ can be computed from $[11,12]$

$$
\Omega_{\mathrm{GW}}\left(k, \eta_{0}\right)=\frac{\mathcal{P}_{T}(k)\left[X^{\prime}\left(k, \eta_{0}\right)\right]^{2}}{24 a_{0}^{2} H_{0}^{2}} .
$$

Using the equations of state computed for different lepton asymmetry values, for which the cosmic trajectory can enter the pion condensed regime, one can estimate the PGW spectrum by using Eqs. (7)-(9). We consider entropy conservation $\left(s a^{3}=\right.$ const) and use the number of degrees of freedom after neutrino decoupling [68] to find the

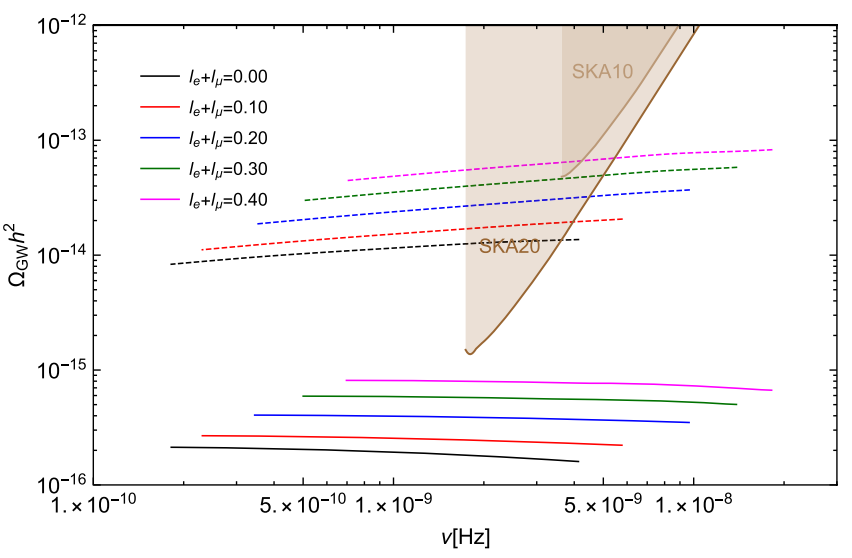

FIG. 4. PGW relic density for different lepton asymmetry values and using the amplitude of scalar perturbation $A_{S}=2.1 \times 10^{-9}$, the scale invariant $n_{T}=0$ (solid lines), and the scale dependent $n_{T}=0.25$ (dashed lines) tensor power spectrum from the upper bound on the tensor to scalar perturbation ratio $r=0.07$ of PLANCK. The future constraints that can be reached by the SKA over 10 and $20 \mathrm{yr}$ of operation are also shown $[69,70]$.

relation between the scale factor and the temperature. The PGW relic spectra are shown in Fig. 4. As the lepton asymmetry increases, so does the amplitude of the spectrum because the entropy, energy, and pressure densities become larger. Moreover, the formation of pion condensation can enhance the PGW due to the change of equation of state. Pulsar timing arrays, as the future such as the Square Kilometre Array (SKA) [69,70], can measure the predicted PGW spectrum especially around the QCD phase transition if it is scale invariant $\left(n_{T}=0\right)$ or blue-tilted $\left(n_{T}>0\right)$. The LISA experiment [71] can also measure such effects at higher frequencies. The lepton asymmetry at BBN time and afterwards is constrained by cosmic microwave background measurements. Since nonvanishing lepton asymmetry and pion condensation before BBN can modify the PGW spectrum, GW observatories with high sensitivity are able to measure these effects in the early Universe.

Impact on the formation of PBHs.-The population of primordial black holes that formed in the early Universe depends on the Hubble rate and the total mass within the Hubble horizon [72-78]. As mentioned earlier, a nonvanishing lepton asymmetry and a pion condensed phase modify the Hubble rate thereby modifying the production of PBHs in a specific range of masses. The horizon mass, defined as $M_{h}=4 \pi / 3 H^{-3} \varepsilon[15,16]$, relates a given temperature in the early Universe to the horizon mass and later on to a typical black hole mass $M_{\mathrm{BH}}$. Figure 5 shows the fraction $f_{\mathrm{PBH}}$ of $\mathrm{PBHs}$ with respect to total cold dark matter (CDM) abundance for different lepton asymmetry cases (see Ref. [34] for the technical details of the calculation). The presence of pion condensation is signaled by a modification of $f_{\mathrm{PBH}}$ at masses larger than one solar mass. 


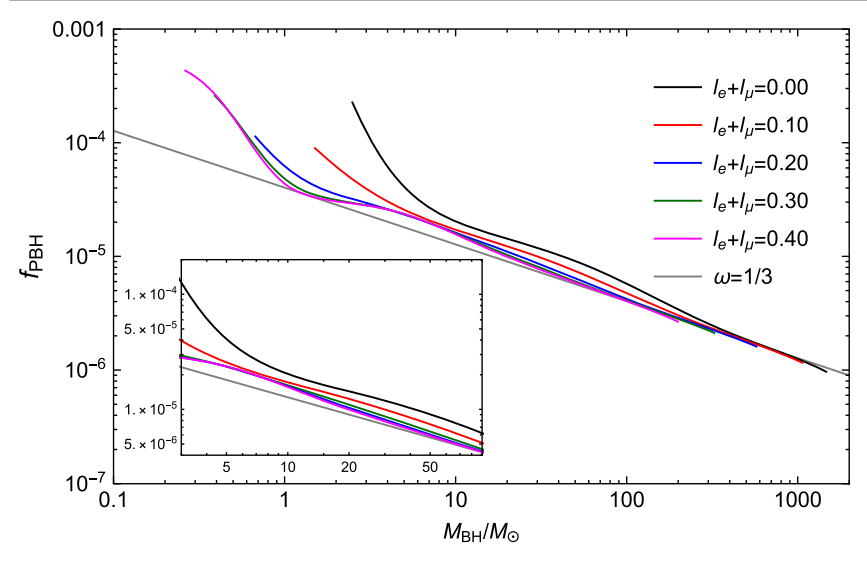

FIG. 5. The fraction of PBHs with respect to PBH masses for different lepton asymmetry values (different colors) assuming the scale invariant Gaussian density perturbation and the value of the density spectral index $n_{M}=0$ [34]. The gray line denotes the result for a purely radiation dominated background fluid.

The parameter $f_{\mathrm{PBH}}$ can be indirectly measured by different experiments. The fraction of PBHs with masses $10^{-6} M_{\odot} \lesssim M_{\mathrm{BH}} \lesssim 10^{3} M_{\odot}$ from some experimental constraints (OGLE, HSC, Caustic, EROS, MACHO) should be $f_{\mathrm{PBH}} \lesssim 0.05[79,82-86]$. The SKA $[69,70]$ and LISA [71] can also indirectly constrain the fraction of PBHs by putting limits on the induced PGWs from curvature perturbation or using GWs produced by coalescing events $[13,87,88]$.

Summary.-The present analysis of cosmic trajectories at nonvanishing lepton flavor asymmetries reveals a simple criterion for the onset of pion condensation in the early Universe-it occurs when the total electron and muon asymmetry parameter is sufficiently large, $\left|l_{e}+l_{\mu}\right| \gtrsim 0.1$. This result does not exhibit large sensitivity to the modeling of pion interactions. Asymmetries beyond this value lead the system deep inside the pion condensed phase, affecting its equation of state considerably. The possible presence of such a Bose-Einstein condensed phase of pions would have significant cosmological implications such as the strong enhancement of the spectrum of PGWs and the change of the fraction of PBHs with mass larger than one solar mass. The experimental signatures of pion condensation from the early Universe can be probed by pulsar timing and GW detectors. The recent $\mathrm{BH}$ merger event of LIGO GW190521 can be from PBHs produced during the pion condensation epoch $[89,90]$.

Pion condensation could also affect big bang nucleosynthesis. If the pion condensed phase is present, spheres of pions and leptons - the pion stars - can form which are stabilized by the high density of neutrinos due to the high lepton chemical potentials $[27,91,92]$. Typical pion star masses will be in the range of a few solar masses when the early Universe leaves the pion condensed phase. The neutrinos will diffuse out of the pion stars on the timescale of weak interactions. The situation is similar to the one for protoneutron stars where neutrinos leave on the timescale of several seconds. Hence, pion stars would decay around the time of BBN. The produced high energy leptons would influence the abundance of primordially produced nuclei, which could be addressed by a modified BBN simulation.

We thank Szabolcs Borsányi for useful correspondence and for providing the data for the Taylor expansion coefficients. We also thank Dietrich Bödeker, Eduardo Fraga, Mauricio Hippert, Pasi Huovinen, Mandy M. Middeldorf-Wygas, Isabel Oldengott, Sebastian Schmalzbauer, Dominik Schwarz, Stephan Wystub, and Yong $\mathrm{Xu}$ for numerous fruitful discussions. V. V. was supported by the Feodor Lynen program of the Alexander von Humboldt foundation and by the U.S. Department of Energy, Office of Science, Office of Nuclear Physics, under Contract No. DE-AC02-05CH11231231. The work of B. B. B., F. C., G. E., F. H., and J. S. is supported by the Deutsche Forschungsgemeinschaft (DFG) through the CRC-TR 211, Project No. 315477589-TRR 211. G. E. also acknowledges support by the DFG Emmy Noether Programme (EN 1064/2-1). The work of F. H. is also supported by the research grant "New Theoretical Tools for Axion Cosmology" under the Supporting TAlent in ReSearch@University of Padova (STARS@UNIPD).

[1] A. Riotto and M. Trodden, Annu. Rev. Nucl. Part. Sci. 49, 35 (1999).

[2] J. M. Cline, arXiv:hep-ph/0609145.

[3] S. Davidson, E. Nardi, and Y. Nir, Phys. Rep. 466, 105 (2008).

[4] M. Flanz, E. A. Paschos, and U. Sarkar, Phys. Lett. B 345, 248 (1995); 384, 487 (1996); 382, 447 (1996).

[5] B. Abbott et al. (LIGO Scientific and Virgo Collaborations), Phys. Rev. Lett. 116, 061102 (2016).

[6] B. Abbott et al. (LIGO Scientific and Virgo Collaborations), Astrophys. J. Lett. 818, L22 (2016).

[7] C. Caprini and D. G. Figueroa, Classical Quantum Gravity 35, 163001 (2018).

[8] L. Grishchuk, Zh. Eksp. Teor. Fiz. 67, 825 (1974) [Sov. Phys. JETP 40, 409 (1975)].

[9] A. A. Starobinsky, JETP Lett. 30, 682 (1979) [JETP Lett. 30, 682 (1979)].

[10] V.F. Mukhanov, H. Feldman, and R. H. Brandenberger, Phys. Rep. 215, 203 (1992).

[11] Y. Watanabe and E. Komatsu, Phys. Rev. D 73, 123515 (2006).

[12] N. Bernal and F. Hajkarim, Phys. Rev. D 100, 063502 (2019).

[13] F. Hajkarim, J. Schaffner-Bielich, S. Wystub, and M. M. Wygas, Phys. Rev. D 99, 103527 (2019).

[14] S. Schettler, T. Boeckel, and J. Schaffner-Bielich, Phys. Rev. D 83, 064030 (2011).

[15] B. J. Carr, Astrophys. J. 201, 1 (1975).

[16] B. J. Carr and S. Hawking, Mon. Not. R. Astron. Soc. 168, 399 (1974).

[17] S. Bird, I. Cholis, J. B. Muñoz, Y. Ali-Haïmoud, M. Kamionkowski, E. D. Kovetz, A. Raccanelli, and A. G. Riess, Phys. Rev. Lett. 116, 201301 (2016). 
[18] M. Y. Khlopov, Res. Astron. Astrophys. 10, 495 (2010).

[19] M. Sasaki, T. Suyama, T. Tanaka, and S. Yokoyama, Classical Quantum Gravity 35, 063001 (2018).

[20] B. Carr, F. Kuhnel, and M. Sandstad, Phys. Rev. D 94, 083504 (2016).

[21] J. A. Harvey and M. S. Turner, Phys. Rev. D 42, 3344 (1990).

[22] I. M. Oldengott and D. J. Schwarz, Europhys. Lett. 119, 29001 (2017).

[23] M. M. Wygas, I. M. Oldengott, D. Bödeker, and D. J. Schwarz, Phys. Rev. Lett. 121, 201302 (2018).

[24] M. M. Middeldorf-Wygas, I. M. Oldengott, D. Bödeker, and D. J. Schwarz, arXiv:2009.00036.

[25] In contrast to Refs. [23,24], where the equation of state is based on Taylor expansion around zero chemical potentials, here we explicitly include a pion-condensed phase at large $\mu_{Q}$ via an effective mass model matched to lattice data.

[26] B. B. Brandt, G. Endrődi, and S. Schmalzbauer, Phys. Rev. D 97, 054514 (2018).

[27] B. B. Brandt, G. Endrődi, E. S. Fraga, M. Hippert, J. Schaffner-Bielich, and S. Schmalzbauer, Phys. Rev. D 98, 094510 (2018).

[28] We discuss the distinction between $\mu_{I}$ and $\mu_{Q}$ in Section II of the Supplemental Material.

[29] H. Abuki, T. Brauner, and H. J. Warringa, Eur. Phys. J. C 64, 123 (2009).

[30] S. Borsányi, Z. Fodor, S. D. Katz, S. Krieg, C. Ratti, and K. Szabó, J. High Energy Phys. 01 (2012) 138.

[31] A. Bazavov et al. (HotQCD Collaboration), Phys. Rev. D 86, 034509 (2012).

[32] O. Savchuk, Y. Bondar, O. Stashko, R. V. Poberezhnyuk, V. Vovchenko, M. I. Gorenstein, and H. Stoecker, Phys. Rev. C 102, 035202 (2020).

[33] D. T. Son and M. A. Stephanov, Phys. Rev. Lett. 86, 592 (2001).

[34] See Supplemental Material at http://link.aps.org/ supplemental/10.1103/PhysRevLett.126.012701 for details on the effective mass model and the comparison to chiral perturbation theory and other model approaches, which includes references to Refs. [35-42], on the lattice simulations and comparisons between the effective mass model and lattice data, which includes references to Refs. [43-46], and for details of the primordial black holes population calculation, which includes references to Refs. [47-52].

[35] H. W. Barz, B. L. Friman, J. Knoll, and H. Schulz, Phys. Rev. D 40, 157 (1989).

[36] P. Adhikari and J. O. Andersen, Phys. Lett. B 804, 135352 (2020).

[37] P. Adhikari and J. O. Andersen, J. High Energy Phys. 06 (2020b) 170.

[38] P. Adhikari and J. O. Andersen, arXiv:2003.12567.

[39] P. Adhikari, J. O. Andersen, and M. A. Mojahed, arXiv:2010.13655.

[40] L.-y. He, M. Jin, and P.-f. Zhuang, Phys. Rev. D 71, 116001 (2005).

[41] P. Adhikari, J. O. Andersen, and P. Kneschke, Phys. Rev. D 98, 074016 (2018).

[42] A. Folkestad and J. O. Andersen, Phys. Rev. D 99, 054006 (2019).
[43] B. B. Brandt and G. Endrődi, Phys. Rev. D 99, 014518 (2019).

[44] S. Borsányi, G. Endrődi, Z. Fodor, A. Jakovác, S. D. Katz, S. Krieg, C. Ratti, and K. K. Szabó, J. High Energy Phys. 11 (2010) 077.

[45] B. B. Brandt and G. Endrődi, Proc. Sci., LATTICE2016 (2016) 039 [arXiv:1611.06758].

[46] M. Mannarelli, Particles 2, 411 (2019).

[47] C. R. Evans and J. S. Coleman, Phys. Rev. Lett. 72, 1782 (1994).

[48] T. Koike, T. Hara, and S. Adachi, Phys. Rev. Lett. 74, 5170 (1995).

[49] I. Musco, J. C. Miller, and A. G. Polnarev, Classical Quantum Gravity 26, 235001 (2009).

[50] I. Musco, J. C. Miller, and L. Rezzolla, Classical Quantum Gravity 22, 1405 (2005).

[51] T. Harada, C.-M. Yoo, and K. Kohri, Phys. Rev. D 88, 084051 (2013); 89, 029903(E) (2014).

[52] A. Escrivà, C. Germani, and R. K. Sheth, Phys. Rev. D 101, 044022 (2020).

[53] K. Olive et al. (Particle Data Group), Chin. Phys. C 38, 090001 (2014).

[54] C. G. Broyden, Math. Comput. 19, 577 (1965).

[55] V. Vovchenko and H. Stoecker, Comput. Phys. Commun. 244, 295 (2019).

[56] I. Affleck and M. Dine, Nucl. Phys. B249, 361 (1985).

[57] M. Stuke, D. J. Schwarz, and G. Starkman, J. Cosmol. Astropart. Phys. 03 (2012) 040.

[58] A. Casas, W. Y. Cheng, and G. Gelmini, Nucl. Phys. B538, 297 (1999).

[59] J. McDonald, Phys. Rev. Lett. 84, 4798 (2000).

[60] K. Abazajian, N. F. Bell, G. M. Fuller, and Y. Y. Y. Wong, Phys. Rev. D 72, 063004 (2005).

[61] K. Ichikawa, M. Kawasaki, and F. Takahashi, Phys. Lett. B 597, 1 (2004).

[62] F. Hajkarim and J. Schaffner-Bielich, Phys. Rev. D 101, 043522 (2020).

[63] K. Saikawa and S. Shirai, J. Cosmol. Astropart. Phys. 05 (2018) 035.

[64] V. Mukhanov, Physical Foundations of Cosmology (Cambridge University Press, Oxford, 2005).

[65] Y. Akrami et al. (Planck Collaboration), Astron. Astrophys. 641, A10 (2020).

[66] N. Aghanim et al. (Planck Collaboration), Astron. Astrophys. 641, A6 (2020).

[67] The initial conditions that we consider at superhorizon scale $(k \ll a H)$ are $X(k, 0)=1$ and $X^{\prime}(k, 0)=0$.

[68] M. Drees, F. Hajkarim, and E. R. Schmitz, J. Cosmol. Astropart. Phys. 06 (2015) 025.

[69] G. Janssen et al., Proc. Sci., AASKA14 (2015) 037 [arXiv:1501.00127].

[70] A. Weltman et al., Pub. Astron. Soc. Aust. 37, e002 (2020).

[71] P. Amaro-Seoane et al. (LISA Collaboration), arXiv: 1702.00786.

[72] C. T. Byrnes, M. Hindmarsh, S. Young, and M. R. S. Hawkins, J. Cosmol. Astropart. Phys. 08 (2018) 041.

[73] P. Widerin and C. Schmid, arXiv:astro-ph/9808142.

[74] J. Sobrinho, P. Augusto, and A. Gonçalves, Mon. Not. R. Astron. Soc. 463, 2348 (2016).

[75] K. Jedamzik, Phys. Rev. D 55, R5871 (1997). 
[76] C. Schmid, D. J. Schwarz, and P. Widerin, Phys. Rev. D 59, 043517 (1999).

[77] J. C. Niemeyer and K. Jedamzik, Phys. Rev. Lett. 80, 5481 (1998).

[78] J. C. Niemeyer and K. Jedamzik, Phys. Rev. D 59, 124013 (1999).

[79] Other experiments can put stronger bounds on $f_{\mathrm{PBH}}$ in a narrower range of masses which we do not consider here (see Refs. [20,80,81]).

[80] B. Carr and F. Kuhnel, arXiv:2006.02838.

[81] B. Carr, K. Kohri, Y. Sendouda, and J. Yokoyama, arXiv: 2002.12778.

[82] H. Niikura et al., Nat. Astron. 3, 524 (2019ba).

[83] H. Niikura, M. Takada, S. Yokoyama, T. Sumi, and S. Masaki, Phys. Rev. D 99, 083503 (2019).

[84] P. Tisserand et al. (EROS-2 Collaboration), Astron. Astrophys. 469, 387 (2007).
[85] C. Alcock et al. (Macho Collaboration), Astrophys. J. Lett. 550, L169 (2001).

[86] M. Oguri, J. M. Diego, N. Kaiser, P. L. Kelly, and T. Broadhurst, Phys. Rev. D 97, 023518 (2018).

[87] S. Wang, T. Terada, and K. Kohri, Phys. Rev. D 99, 103531 (2019); 101, 069901(E) (2020).

[88] N. Bartolo, V. De Luca, G. Franciolini, M. Peloso, D. Racco, and A. Riotto, Phys. Rev. D 99, 103521 (2019).

[89] R. Abbott et al. (LIGO Scientific and Virgo Collaborations), Astrophys. J. Lett. 900, L13 (2020).

[90] R. Abbott et al. (LIGO Scientific and Virgo Collaborations), Phys. Rev. Lett. 125, 101102 (2020).

[91] S. Carignano, L. Lepori, A. Mammarella, M. Mannarelli, and G. Pagliaroli, Eur. Phys. J. A 53, 35 (2017).

[92] J. O. Andersen and P. Kneschke, arXiv:1807.08951. 